\title{
On the Distance between Three Arbitrary Points
}

\author{
Parin Chaipunya and Poom Kumam \\ Department of Mathematics, Faculty of Science, King Mongkut's University of Technology Thonburi, Bangkok 10140, Thailand
}

Correspondence should be addressed to Poom Kumam; poom.kum@kmutt.ac.th

Received 25 March 2013; Accepted 1 August 2013

Academic Editor: Satit Saejung

Copyright (C) 2013 P. Chaipunya and P. Kumam. This is an open access article distributed under the Creative Commons Attribution License, which permits unrestricted use, distribution, and reproduction in any medium, provided the original work is properly cited.

We point out some equivalence between the results in (Sedghi et al., 2012) and (Khamsi, 2010). Then, we introduce the notion of a general distance between three arbitrary points and study some of its properties. In the final section, some fixed point results are proposed.

\section{Introduction}

The literature of a distance for any triple of points in a space was first considered during the sixties by Gähler $[1,2]$. It is known as a 2-metric, the concept of which was later extended by Dhage [3] into a $D$-metric. Both notions are in no easy ways related to the classical concept of a metric. This led to the $G$-metric due to Mustafa and Sims [4].

On the other hands, Sedghi et al. [5] had introduced the notion of a $D^{*}$-metric, which has later been generalized by Sedghi et al. [6] into an $S$-metric.

These developments confirm that this kind of measurement is recently of many mathematicians' interests. One of the area that exploited these establishments largely is the fixed point theory, especially the ones involving some generalized contractions; see, for example, Sedghi et al. [5], Sedghi et al. [6], Mustafa et al. [7], Mustafa and Sims [8], Aydi et al. [9], Alghamdi and Karapinar [10], Chandok et al. [11], and Abbas et al. [12].

In this present paper, we divide our interests into three parts. Firstly, we give a remark on the existing fixed point result endowed in a $S$-metric space. Secondly, we propose and study a very general principle in measuring the distance between three arbitrary points called a $g-3 p s$. Thirdly, we construct some fixed point theorems by utilizing the $g-3 p s$ and its properties.

\section{Preliminaries}

This section is devoted to the recollection of important definitions and lemmas. We start with a sequence of definitions of $G_{-}, D^{*}$-, and $S$-metric spaces.

Definition 1 (see [4]). Let $X$ be a nonempty set. A function $G: X \times X \times X \rightarrow \mathbb{R}_{+}$is said to be a $G$-metric if the following conditions are satisfied:

(1) for $x, y, z \in X, G(x, y, z)=0$ if $x=y=z$;

(2) for $x, y \in X$ with $x \neq y, G(x, x, y)>0$;

(3) for $x, y, z \in X$ with $y \neq z, G(x, x, y) \leq G(x, y, z)$;

(4) for $x, y, z \in X, G(x, y, z)=G(\pi(x, y, z))$, where $\pi(x, y, z)$ is any permutation of $(x, y, z) \in X \times X \times X$;

(5) for $x, y, z, v \in X, G(x, y, z) \leq G(x, v, v)+G(v, y, z)$.

The pair $(X, G)$ is called a $G$-metric space. Moreover, if $G(x, x, y)=G(y, y, x)$ for all $x, y \in X$, then $G$ is said to be symmetric.

Definition 2 (see [5]). Let $X$ be a nonempty set. A function $D^{*}: X \times X \times X \rightarrow \mathbb{R}_{+}$is said to be a $D^{*}$-metric if the following conditions are satisfied:

(1) for $x, y, z \in X, D^{*}(x, y, z)=0$ if and only if $x=y=$ $z$ 
(2) for $x, y, z \in X, D^{*}(x, y, z)=D^{*}(\pi(x, y, z))$, where $\pi(x, y, z)$ is any permutation of $(x, y, z) \in X \times X \times X$;

(3) for $x, y, z, v \in X, D^{*}(x, y, z) \leq D^{*}(x, y, v)+D^{*}(v$, $z, z)$.

The pair $\left(X, D^{*}\right)$ is called a $D^{*}$-metric space.

Definition 3 (see [6]). Let $X$ be a nonempty set. A function $S: X \times X \times X \rightarrow \mathbb{R}_{+}$is said to be a $S$-metric if the following conditions are satisfied:

(1) for $x, y, z \in X, S(x, y, z)=0$ if and only if $x=y=z$;

(2) for $x, y, z, v \in X, S(x, y, z) \leq S(x, x, v)+S(y, y, v)+$ $S(z, z, v)$.

The pair $(X, S)$ is called a $S$-metric space.

Lemma 4 (see [6]). Let $(X, S)$ be a S-metric space, then $S(x, x, y)=S(y, y, x)$ for all $x, y \in X$.

It can be seen that each symmetric $G$-metric is a $D^{*}$ metric and that each $D^{*}$-metric is a $S$-metric. In case of nonsymmetric $G$-metric, the concepts of $G$-metric and $S$-metric are independent.

Definition 5 (see [6]). Let $(X, S)$ be a $S$-metric space. For $r>0$ and $x \in X$ we define the open ball $B_{S}(x ; r)$ as follows:

$$
B_{S}(x ; r)=\{y \in X ; S(x, x, y)<r\} .
$$

As in [6], one may consider the topology $\tau$ for $X$ which is generated from the base containing all open balls in $X$. Some concepts are also introduced.

Definition 6 (see [6]). Let $(X, S)$ be a $S$-metric space. A sequence $\left(x_{n}\right)$ in $X$ is called

(i) Cauchy if for any $\epsilon>0$, we may find $N \in \mathbb{N}$ such that

$$
n \in \mathbb{N}, n \geq N \Longrightarrow S\left(x_{m}, x_{m}, x_{n}\right)<\epsilon
$$

(ii) convergent if there is a point $x \in X$ in which

$$
\lim _{n \rightarrow \infty} S\left(x, x, x_{n}\right)=0 \text {. }
$$

Moreover, if every Cauchy sequence in $X$ is also convergent, $X$ is said to be complete.

\section{A S-Metric Space as a Metric Type Space}

In this section, we shall be giving a small remark on a fixed point theorem due to [6]. According to [6], a self-operator $f$ on a $S$-metric space $(X, S)$ is called a contraction if it satisfies the following inequality:

$$
S(f x, f x, f y) \leq \lambda S(x, x, y),
$$

for all $x, y \in X$, where $0 \leq \lambda<1$. The following result was introduced subsequently.
Theorem 7 (see [6]). Let $(X, S)$ be a complete S-metric space and let $f$ be a contraction on $X$. Then, $f$ has a unique fixed point.

To expedite our remark, we shall first recall the notion of a metric type space, which was introduced by Khamsi in [13].

Definition 8 (see [13]). Let $X$ be a nonempty set and let $D: X \times X \rightarrow \mathbb{R}_{+}$be a function satisfying the following conditions:

(1) for $x, y \in X, D(x, y)=0$ if and only if $x=y$;

(2) for $x, y \in X, D(x, y)=D(y, x)$;

(3) there exists a constant $K>0$ such that for $x, y, z_{1}$, $z_{2}, \ldots, z_{n} \in X$

$$
D(x, y) \leq K\left[D\left(x, z_{1}\right)+D\left(z_{1}, z_{2}\right)+\cdots+D\left(z_{n}, y\right)\right] \text {. }
$$

The triple $(X, D, K)$ is called a metric type space.

In particular, if $K \leq 1$, then $(X, D)$ is a metric space. A self-mapping operator $f$ on a metric type space $(X, D, K)$ is called Lipschizian if there exists $\lambda \geq 0$ such that

$$
D(f x, f y) \leq \lambda D(x, y),
$$

for all $x, y \in X$. The smallest $\lambda>0$ satisfying such condition is denoted by $\operatorname{Lip}(f)$. Moreover, the following fixed point theorem was proposed.

Theorem 9 (see [13]). Let $(X, D, K)$ be a complete metric type space and let $f: X \rightarrow X$ be an operator such that the composition $f^{n}$ is Lipschizian for each $n \in \mathcal{N}$ and $\sum_{n \in \mathbb{N}} \operatorname{Lip}\left(f^{n}\right)<\infty$. Then, $f$ has a unique fixed point.

It follows that if $f$ is a Lipschizian operator with $\operatorname{Lip}(f)<$ 1 , then $f$ has a unique fixed point.

Now, let $(X, S)$ be a $S$-metric space. Suppose that a function $D: X \times X \rightarrow \mathbb{R}_{+}$is given by

$$
D_{S}(x, y):=S(x, x, y), \quad \forall x, y \in X,
$$

for $x, y \in X$, it is obvious that $D(x, y)=0$ if and only if $x=y$ and $D(x, y)=D(y, x)$ for all $x, y \in X$. Now, observe for each $z_{1}, z_{2}, \ldots, z_{n} \in X$ that

$$
\begin{aligned}
D_{S}(x, y)= & S(x, x, y) \\
\leq & 2 S\left(x, x, z_{1}\right)+S\left(y, y, z_{1}\right) \\
= & 2 D_{S}\left(x, z_{1}\right)+D_{S}\left(z_{1}, y\right) \\
\leq & 2 D_{S}\left(x, z_{1}\right)+2 D_{S}\left(z_{1}, z_{2}\right)+D_{S}\left(z_{2}, y\right) \\
\vdots & \\
\leq & 2 D_{S}\left(x, z_{1}\right)+2 D_{S}\left(z_{1}, z_{2}\right) \\
& +\cdots+2 D_{S}\left(z_{n-1}, z_{n}\right)+D_{S}\left(z_{n}, y\right) \\
\leq & 2\left[D_{S}\left(x, z_{1}\right)+D_{S}\left(z_{1}, z_{2}\right)\right. \\
& \left.\quad+\cdots+D_{S}\left(z_{n-1}, z_{n}\right)+D_{S}\left(z_{n}, y\right)\right] .
\end{aligned}
$$


Thus, $\left(X, D_{S}, 2\right)$ is a metric type space. Moreover, the balls $B_{S}(x ; r)$ and $B_{D_{S}}(x ; r)$ coincide.

Notice that we may rewrite the inequality (4) as follows:

$$
D_{S}(f x, f y) \leq \lambda D_{S}(x, y),
$$

for all $x, y \in X$ with the same $\lambda$. Also notice that the definition of Cauchyness, convergence and completeness in a $S$-metric space $(X, S)$ may be rewriten in terms of metric type spaces. So, these notions are transferred to the corresponding metric type space $\left(X, D_{S}, 2\right)$.

Now, if $f$ is an operator satisfying (9), then each $f^{n}$, where $n \in \mathbb{N}$, is Lipschizian with $\operatorname{Lip}\left(f^{n}\right)=\lambda^{n}$. Therefore, Theorem 7 is obtained via Theorem 9 .

Even though we set a new condition for an operator $f$, where $0 \leq \lambda<1$, to be

$$
S(f x, f y, f z) \leq \lambda S(x, y, z)
$$

for each $x, y, z \in X$, the unique fixed point can still be obtained via Theorem 9, anyway. Note that not only the mentioned theorem, but also many theorems in the literature may be proved via this concept in metric type spaces. We shall give some results which seem more general than the setting of Theorem 7 but however equivalent.

Beforehand, we give the following useful lemma without proof since it is straight forward.

Lemma 10. Let $(X, S)$ be a S-metric space and let $x, y \in X$. Then, the following inequalities hold:

(i) $S(x, y, y) \leq S(x, x, y)=S(y, y, x)$;

(ii) $S(x, y, x) \leq S(x, x, y)=S(y, y, x)$.

Theorem 11. Let $(X, S)$ be a complete S-metric space and let $f: X \rightarrow X$ be an operator such that there exists a sequence $\left(\lambda_{n}\right)$ of nonnegative reals satisfying the condition:

$$
S(f x, f y, f z) \leq \sum_{n \in \mathbb{N}} \lambda_{n} S\left(\pi_{n}(x, y, z)\right),
$$

for all $x, y, z \in X$, where $\Lambda:=\sum_{n \in \mathbb{N}} \lambda_{n}<1$ and for each $n \in \mathbb{N}$, $\pi_{n}$ is a fixed permutation in $X^{3}$. Then, $f$ has a unique fixed point.

Proof. We shall show that (11) implies that $f$ is Lipschizian with $\operatorname{Lip}(f)<1$ in metric type space $\left(X, D_{S}, 2\right)$. For each $x, y \in X$, it is easy to verify that $S\left(\pi_{n}(x, x, y)\right) \leq S(x, x, y)$ no matter which permutations are defined. Thus, we obtain that

$$
\begin{aligned}
D_{S}(f x, f y) & =S(f x, f x, f y) \\
& \leq \sum_{n \in \mathbb{N}} \lambda_{n} S\left(\pi_{n}(x, x, y)\right) \\
& \leq \Lambda S(x, x, y) \\
& =\Lambda D_{S}(x, y) .
\end{aligned}
$$
result.

\section{A General Distance between Three Arbitrary Points}

In this section, we shall be dealing with a new concept of a general distance between three arbitrary points (or $g-3 p s$ ).

To be able to define the $g-3 p s$, we first consider a nonempty set $X$ together with a function $g: X \times X \times X \rightarrow \mathbb{R}_{+}$ for which $g(x, y, z)=0$ if and only if $x=y=z$. Given $x \in X$ and $r>0$, we define an open ball in the usual sense:

$$
B_{g}(x ; r):=\{y \in X ; g(x, x, y)<r\} .
$$

To be natural, we say that a subset $A \subset X$ is bounded if $\sup _{x, y, z \in A} g(x, y, z)<\infty$. Certainly, the assertion " $g(x, y, z)=0$ if and only if $x=y=z$ " is not enough to guarantee that open balls in $X$ are bounded. We shall illustrate in the following.

Example 12. Let $X:=[0,1]$ and let $g: X \times X \times X \rightarrow \mathbb{R}_{+}$be a function given by

$$
g(x, y, z):= \begin{cases}0, & \text { if } x=y=z, \\ \left|\left(\frac{x+y}{2}\right)-z\right|, & \text { if } x, y \in \mathbb{Q}_{\mathbb{Q}} \cap X \\ \left|\left(\frac{x+y}{2}\right)-z\right|, & \text { but } z \in \mathbb{Q}_{C} \cap X, \\ \frac{1}{|((x+y) / 2)-z|}, & \text { if } x, y \in \mathbb{Q}_{C} \cap X \\ \frac{1}{1}, y, z \in \mathbb{Q} \cap X & \text { and }[x \neq y \text { or } y \neq z], \\ \frac{|((x+y) / 2)-z|,}{} & \text { if } x, y, z \in \mathbb{Q}_{C} \cap X \\ & \text { and }[x \neq y \text { or } y \neq z], \\ 1, & \text { otherwise. }\end{cases}
$$

It is clear that $g(x, y, z)=0$ if and only if $x=y=z$.

Let $x \in X$ and let $r>0$. Note that if $x \in \mathbb{Q} \cap X$, then

$$
\mathbb{Q}_{C} \cap(x-r, x+r) \cap X \subset B_{g}(x ; r) .
$$

Let $\left(z_{n}\right)$ be a sequence in $\mathbb{Q}_{C} \cap(x-r, x+r) \cap X$ such that $\mid x-$ $z_{n} \mid<r /(1+n r)$ for each $n \in \mathbb{N}$. Since $\left|z_{n}-z_{n+1}\right|<2 r /(1+n r)$, we have $g\left(z_{n}, z_{n}, z_{n+1}\right)>(1 / 2 r)+(n / 2)$ for all $n \in \mathbb{N}$. The same conclusion can be deduced also when $x \in \mathbb{Q}_{C}$. Therefore, $B_{g}(x ; r)$ is not bounded at each $x \in X$ and $r>0$.

This is not quite natural and does not meet the requirements we would like to have. So, we may add one more assumption at this stage and define the $g-3 p s$ space as follows.

Definition 13. Let $X$ be a nonempty set. A function $g: X \times X \times$ $X \rightarrow \mathbb{R}_{+}$is said to be a $g-3 p s$ if the following conditions are satisfied:

(g1) for $x, y, z \in X, g(x, y, z)=0$ if and only if $x=y=z$;

(g2) there exists some $r_{0}>0$ such that the balls $B_{g}\left(x ; r_{0}\right)$ are bounded for all $x \in X$.

The pair $(X, g)$ is called a $g-3 p s$ space. 
Next, we shall give a characterization of a $g-3 p s$ space.

Lemma 14. Let $X$ be a nonempty set and let $g: X \times X \times X \rightarrow$ $\mathbb{R}_{+}$be a function satisfying ( $\left.g 1\right)$. Then, the following are equivalent:

(i) g satisfies ( $g 2$ );

(ii) there exist some constants $\delta, \eta>0$ such that for any $x, u, v, w \in X$, one has

$$
g(x, x, u)+g(x, x, v)+g(x, x, w)<\delta \Longrightarrow g(u, v, w)<\eta
$$

Proof. [(i) $\Rightarrow$ (ii)] Assume that (i) holds. Set $\delta:=r / 2$ and let $x, u, v, w \in X$ arbitrarily. If $g(x, x, u)+g(x, x, v)+$ $g(x, x, w)<\delta$, then $u, v, w \in B_{g}(x ; r)$. Thus, setting $\eta:=$ $1+\sup _{x \in X} \sup _{a, b, c \in B_{g}(x ; r)} g(a, b, c)<\infty$ and it follows that $g(u, v, w)<\eta$.

[(ii) $\Rightarrow$ (i)] Assume that (ii) holds. Let $x \in X$ and suppose that $u, v, w \in B_{g}(x, \delta / 3)$. Therefore, we have

$$
g(x, x, u)+g(x, x, v)+g(x, x, w)<\delta .
$$

From (ii), we obtain that $g(u, v, w)<\eta$. Thus, $\sup _{a, b, c \in B_{g}(X ; \delta / 3)} g(a, b, c) \leq \eta$. Since $x \in X$ is arbitrary, the balls $B_{g}(x ; \delta / 3)$ are bounded for every $x \in X$.

Remark 15. Suppose that $(X, G)$ is a $G$-metric space and $x, u, v, w \in X$. Then, we have

$$
\begin{aligned}
G(u, v, w) & \leq G(u, x, x)+G(x, v, w) \\
& =G(x, x, u)+G(v, x, w) \\
& \leq G(x, x, u)+G(v, x, x)+G(x, x, w) \\
& =G(x, x, u)+G(x, x, v)+G(x, x, w) .
\end{aligned}
$$

Thus, we can choose any $\delta>0$ and let $\eta:=\delta$ to fulfill the assumptions in Lemma 14 . Hence, every $G$-metric space is in turn a $g-3$ s space.

Remark 16. Suppose that $(X, S)$ is a $S$-metric space and $x, u, v, w \in X$. Then, we have

$$
\begin{aligned}
S(u, v, w) & \leq S(u, u, x)+S(v, v, x)+S(w, w, x) \\
& =S(x, x, u)+S(x, x, v)+S(x, x, w) .
\end{aligned}
$$

The same argument is to be considered as in the previous remark. So, a $S$-metric space is a $g-3 p s$ space. This immediately implies that a $D^{*}$-metric space is also a $g-3 p s$ space.

Denoted by $\mathcal{U}$ the family of all open balls in $X$. Throughout this paper, we shall assume that $\mathscr{T}:=\mathscr{T}(\mathcal{U})$ represents the topology having $\mathcal{U}$ as its subbase. Also, we write $\mathcal{U}^{*}$ to denote the base generated by $\mathcal{U}$.

Remark 17. The topology here is defined using different idea from those given in symmetric spaces or in semimetric spaces (see e.g., [14-17]).
Proposition 18. The topology $\mathscr{T}$ for a $g-3$ ps space $(X, g)$ is $T_{1}$-separable.

Proof. Let $x, y \in X$ with $x \neq y$. So, we have $g(x, x, y)=r_{1}$ and $g(y, y, x)=r_{2}$, for some $r_{1}, r_{2}>0$. Observe that $y \notin$ $B_{g}\left(x ; r_{1} / 2\right)$ and $x \notin B_{g}\left(y ; r_{2} / 2\right)$. The desired result is then followed.

We shall now explicate an example of a $g-3 p s$ space. In particular, this next example will even show that a $g-3 p s$ space is no need to be $T_{2}$-separable.

Example 19. Let $X=[0,1]$ and define a function $g: X \times X \times$ $X \rightarrow \mathbb{R}_{+}$in the following:

$$
g(x, y, z):= \begin{cases}0, & \text { if } x=y=z \\ z, & \text { if } x=y \neq z, z \neq 0 \\ 1, & \text { otherwise. }\end{cases}
$$

It is clear that any subset in this space is always bounded. Hence, $(X, g)$ is a $g-3 p s$ space. Observe that for $x \in X$ and $r>0$, we have

$$
B_{g}(x ; r)= \begin{cases}X \cap[\{x\} \cup(0, r)], & \text { if } r \leq 1, \\ X, & \text { if } r>1\end{cases}
$$

Therefore, any two balls intersect one another, implying that $X$ is not $T_{2}$-separable.

We next introduce a new concept of convergence and compare it with the classical topological ones.

Definition 20. Let $(X, g)$ be a $g-3 p$ s space. A sequence $\left(x_{n}\right)$ in $X$ is said to be

(1) Cauchy if for any $\epsilon>0$, there exists $N \in \mathbb{N}$ such that

$$
m, n \geq N \Longrightarrow g\left(x_{m}, x_{m}, x_{n}\right)<\epsilon ;
$$

(2) g-convergent if we can find a point $x \in X$ in which for any $\epsilon>0$, there exists $N \in \mathbb{N}$ satisfying

$$
n \geq N \Longrightarrow g\left(x, x, x_{n}\right)<\epsilon .
$$

In this case, we say that $\left(x_{n}\right) g$-converges to $x$ and write $x_{n} \stackrel{g}{\rightarrow} x$.

Remark 21. Given a $g-3 p$ space $(X, g)$, a sequence $\left(x_{n}\right)$ in $X g$-converges to $x \in X$ if and only if for any set $U \in \mathcal{U}$ with $U \ni x$, there exists $N \in \mathbb{N}$ such that

$$
n \geq N \Longrightarrow x_{n} \in U
$$

Lemma 22. Let $(X, g)$ be a $g-3$ ps space and let $\left(x_{n}\right)$ be a sequence in $X$. Then, the following are equivalent:

(i) $\left(x_{n}\right)$ converges to $x \in X$ in the topology $\mathscr{T}$;

(ii) for any neighborhood $V \in \mathscr{T}$ of $x$, on can find $N \in \mathbb{N}$ such that

$$
n \geq N \Longrightarrow x_{n} \in V
$$


(iii) for any set $U \in \mathcal{U}^{*}$ containing $x$, we can find $N \in \mathbb{N}$ such that

$$
n \geq N \Longrightarrow x_{n} \in U
$$

Proof. (i) $\Leftrightarrow$ (ii) is by definition. So, we only need to show that (ii) $\Leftrightarrow$ (iii).

[(ii) $\Rightarrow$ (iii)] Since $\mathscr{U}^{*} \in \mathscr{T}$, we again apply (ii) to obtain our desired result.

[(iii) $\Rightarrow$ (ii)] By the definition of $\mathscr{T}$, for every $z \in F \in \mathscr{T}$, we can find $E \in \mathscr{U}^{*}$ in which $x \in E \subset F$. Suppose that $V \in \mathscr{T}$ is a neighborhood of $x$, then we can find some $U_{0} \in \mathcal{U}^{*}$ such that $x \in U_{0} \subset V$. Applying (ii), we obtain that $x_{n} \in V$ for every $n \geq N$ for some fixed $N \in \mathbb{N}$.

We conclude the following lemma immediately from Remark 21 and Lemma 22.

Lemma 23. Let $(X, g)$ be a $g-3$ ps space and let $\left(x_{n}\right)$ be a sequence in $X$. If $\left(x_{n}\right)$ converges to some point $x \in X$ in the topology $\mathscr{T}$, then it also $g$-converges to $x$.

This lemma shows that the concept of $g$-convergence is weaker than convergence in topology. However, in case when $(X, g)$ is a $G$-metric space or when $(X, g)$ is a $S$-metric space, the two concepts coincide. Along this paper, we shall deal with this new kind of convergence, rather than those topological ones.

Definition 24. A $g-3 p s$ space $(X, g)$ is called

(i) $g$-Hausdorff if every $g$-convergent sequence $g$ converges to at most one point;

(ii) $\Sigma$-complete if every sequence $\left(x_{n}\right)$ satisfying $\sum_{n \in \mathbb{N}} g\left(x_{n}, x_{n}, x_{n+1}\right)<\infty g$-converges;

(iii) Cauchy-complete if every Cauchy sequence $g$ converges.

Remark 25. A $g-3 p s$ space $(X, g)$ is $g$-Hausdorff if and only if for any two distinct points $x, y \in X$, there exist two disjoint sets $U, V \in \mathcal{U}$ such that $U \ni x$ and $V \ni y$.

\section{Some Fixed Point Theorems}

Under this section, we propose some fixed point theorems in the framework of a $g-3 p s$ space.

We first introduce a lemma which will be used in our main theorems.

Lemma 26. Let $(X, g)$ be a $g-3$ ps space. Suppose that $f$ : $X \rightarrow X$ be an operator such that

$$
g(f x, f y, z) \leq \lambda g(\pi(x, y, z))
$$

for all $x, y, z \in X$, where $0 \leq \lambda<1$ and $\pi$ is a fixed permutation on $X^{3}$. Then, the following hold for every $x \in X$ :

(i) $\sum_{n \in \mathbb{N}} g\left(f^{n} x, f^{n} x, f^{n+1} x\right)<\infty$;

(ii) $\sum_{n \in \mathbb{N}} g\left(f^{n+1} x, f^{n+1} x, f^{n} x\right)<\infty$.
Proof. Let $x \in X$ be arbitrary. We shall consider the permutation $\pi$ case-by-case.

(i) Case $I: \pi(x, y, z):=(x, y, z)$ or $\pi(x, y, z):=(y, x, z)$. Observe that

$$
\begin{aligned}
g\left(f^{n} x, f^{n} x, f^{n+1} x\right) & \leq \lambda g\left(f^{n-1} x, f^{n-1} x, f^{n} x\right) \\
& \leq \lambda^{2} g\left(f^{n-2} x, f^{n-2} x, f^{n-1} x\right) \\
& \vdots \\
& \leq \lambda^{n} g(x, x, f x) .
\end{aligned}
$$

(ii) Case II: $\pi(x, y, z):=(x, z, y)$.

Observe that

$$
\begin{aligned}
g\left(f^{n} x, f^{n} x, f^{n+1} x\right) & \leq \lambda g\left(f^{n-1} x, f^{n} x, f^{n-1} x\right) \\
& \leq \lambda^{2} g\left(f^{n-2} x, f^{n-2} x, f^{n-1} x\right) \\
& \vdots \\
& \leq \lambda^{n} \Gamma_{1},
\end{aligned}
$$

where $\infty>\Gamma_{1} \geq \max \{g(x, x, f x), g(x, f x, x)\}$.

(iii) Case III: $\pi(x, y, z):=(y, z, x)$.

Observe that

$$
\begin{aligned}
g\left(f^{n} x, f^{n} x, f^{n+1} x\right) & \leq \lambda g\left(f^{n-1} x, f^{n} x, f^{n-1} x\right) \\
& \leq \lambda^{2} g\left(f^{n-1} x, f^{n-2} x, f^{n-2} x\right) \\
& \leq \lambda^{3} g\left(f^{n-3} x, f^{n-3} x, f^{n-2} x\right) \\
& \vdots \\
& \leq \lambda^{n} \Gamma_{2},
\end{aligned}
$$

where $\infty>\Gamma_{2} \geq \max \{g(x, x, f x), f(x, f x, x), f(f x, x, x)\}$.

(iv) Case $I V: \pi(x, y, z):=(z, x, y)$ or $\pi(x, y, z):=$ $(z, y, x)$.

Observe that

$$
\begin{aligned}
g\left(f^{n} x, f^{n} x, f^{n+1} x\right) & \leq \lambda g\left(f^{n} x, f^{n-1} x, f^{n-1} x\right) \\
& \leq \lambda^{2} g\left(f^{n-2} x, f^{n-2} x, f^{n-1} x\right) \\
& \vdots \\
& \leq \lambda^{n} \Gamma_{1} .
\end{aligned}
$$

In each case, we may conclude that

$$
\sum_{n \in \mathbb{N}} g\left(f^{n} x, f^{n} x, f^{n+1} x\right) \leq \Gamma_{2} \sum_{n \in \mathbb{N}} \lambda^{n}<\infty .
$$

Similarly, we may prove that

$$
\sum_{n \in \mathbb{N}} g\left(f^{n+1} x, f^{n+1} x, f^{n} x\right) \leq \Gamma_{3} \sum_{n \in \mathbb{N}} \lambda^{n}<\infty,
$$

where $\infty>\Gamma_{3} \geq \max \{g(f x, f x, x), g(f x, x, f x), g(x, f x$, $f x)\}$. 
Now, we consider our fixed point results, which exploited the above lemma.

Theorem 27. Let $(X, g)$ be a g-Hausdorff $\sum$-complete $g-3 p s$ space. Suppose that $f: X \rightarrow X$ is a g-sequentially continuous operator (i.e., $x_{n} \stackrel{g}{\rightarrow} x \Rightarrow f x_{n} \stackrel{g}{\rightarrow} f x$, for every sequence $\left(x_{n}\right)$ in $X)$ satisfying

$$
g(f x, f y, z) \leq \lambda g(\pi(x, y, z)),
$$

for all $x, y, z \in X$, where $0 \leq \lambda<1$ and $\pi$ is a fixed permutation on $X^{3}$. Then, $f$ has exactly one fixed point.

Proof. Let $x \in X$ be arbitrary. By (i) in Lemma 26, we have

$$
\sum_{n \in \mathbb{N}} g\left(f^{n} x, f^{n} x, f^{n+1} x\right)<\infty
$$

Since $X$ is $\sum$-complete, $\left(f^{n} x\right) g$-converges to some point $x_{*} \in X$. Now, since $f$ is $g$-sequentially continuous, $f f^{n} x=$ $f^{n+1} x \stackrel{g}{\rightarrow} f x_{*}$. Since $X$ is $g$-Hausdorff, we have $f x_{*}=x_{*}$. that

$$
g\left(x_{*}, x_{*}, y_{*}\right)=g\left(f x_{*}, f x_{*}, f y_{*}\right) \leq \lambda g\left(x_{*}, x_{*}, y_{*}\right) .
$$

The only possibility of the value of $g\left(x_{*}, x_{*}, y_{*}\right)$ allows us to conclude that $x_{*}=y_{*}$. So, the theorem is proved.

Theorem 28. Let $(X, g)$ be a g-Hausdorff Cauchy-complete $g-3$ ss space. Suppose that $f: X \rightarrow X$ is a $g$-sequentially continuous operator satisfying

$$
g(f x, f y, f z) \leq \lambda g(\pi(x, y, z))
$$

for all $x, y, z \in X$, where $0 \leq \lambda \leq \delta / 3 \eta$ and $\delta, \eta>0$ are given as in Lemma 14. Then, $f$ has exactly one fixed point.

Proof. Let $x \in X$. From Lemma 26, we also have $g\left(f^{n} x, f^{n} x\right.$, $\left.f^{n+1} x\right) \rightarrow 0$ and $g\left(f^{n+1} x, f^{n+1} x, f^{n} x\right) \rightarrow 0$. So, we may choose $N \in \mathbb{N}$ such that

$$
\begin{gathered}
g\left(f^{N} x, f^{N} x, f^{N+1} x\right)<\frac{\delta}{3}, \\
g\left(f^{N+1} x, f^{N+1} x, f^{N} x\right)<\frac{\delta}{3} .
\end{gathered}
$$

Consequently, we obtain that

$$
g\left(f^{N} x, f^{N} x, f^{N+1} x\right)<\eta .
$$

We will show that $g\left(f^{N} x, f^{N} x, f^{N+n} x\right)<\eta$ for all $n \in \mathbb{N}$ via the mathematical induction. Now, we assume that

$$
g\left(f^{N} x, f^{N} x, f^{N+n_{0}} x\right)<\eta
$$

for some $n_{0} \in \mathbb{N}$. Then, observe that

$$
\begin{aligned}
& 2 g\left(f^{N+1} x, f^{N+1} x, f^{N} x\right)+g\left(f^{N+1} x, f^{N+1} x, f^{N+n_{0}+1} x\right) \\
& \quad \leq 2 g\left(f^{N+1} x, f^{N+1} x, f^{N} x\right)+\lambda g\left(f^{N} x, f^{N} x, f^{N+n_{0}} x\right) \\
& \quad<2 g\left(f^{N+1} x, f^{N+1} x, f^{N} x\right)+\lambda \eta \\
& \quad<\delta .
\end{aligned}
$$

Hence, from Lemma 14, we have $g\left(f^{N} x, f^{N} x, f^{N+n_{0}+1} x\right)<\eta$. Therefore, we have $g\left(f^{N} x, f^{N} x, f^{N+n} x\right)<\eta$ for all $n \in \mathbb{N}$.

Let $\epsilon>0$ be given and let $m, n \in \mathbb{N}$ with $N \leq m<n$. Thus, we may write

$$
\begin{aligned}
& m=N+\ell+p, \\
& n=N+\ell+q,
\end{aligned}
$$

for some $\ell, p, q \in \mathbb{N}$. Note that $p<q$. It follows that

$$
\begin{aligned}
g\left(f^{m} x, f^{m} x, f^{n} x\right) & =g\left(f^{N+\ell+p} x, f^{N+\ell+p} x, f^{N+\ell+q} x\right) \\
& \leq \lambda g\left(f^{N+\ell+p-1} x, f^{N+\ell+p-1} x,\right. \\
& \left.f^{N+\ell+q-1} x\right) \\
& \vdots \\
& \leq \lambda^{\ell} \lambda^{p} g\left(f^{N} x, f^{N} x, f^{N+q-p} x\right) \\
& <\lambda^{\ell} \lambda^{p} \eta .
\end{aligned}
$$

Since $\lambda \leq \delta / 3 \eta<1$, if $M:=N+\ell$ is chosen large enough so that $\lambda^{\ell} \eta<\epsilon$, then we ended up with $g\left(f^{m} x, f^{m} x, f^{n} x\right)<$ $\epsilon$. Therefore, $\left(f^{n} x\right)$ is Cauchy. By mean of the Cauchycompleteness of $X$, it converges to some point $x_{*} \in X$. Since $f$ is $g$-sequentially continuous, we have $f f^{n} x \stackrel{g}{\rightarrow} f x_{*}$. Moreover, since $X$ is $g$-Hausdorff, $f x_{*}=x_{*}$.

Assume that $y_{*} \in X$ is also a fixed point of $f$. Observe that

$$
g\left(x_{*}, x_{*}, y_{*}\right)=g\left(f x_{*}, f x_{*}, f y_{*}\right) \leq \lambda g\left(x_{*}, x_{*}, y_{*}\right) .
$$

This implies that $x_{*}=y_{*}$. Therefore, the uniqueness is proved.

Example 29. Let $X=[0,1]$ and define a function $g: X \times X \times$ $X \rightarrow \mathbb{R}_{+}$by

$$
g(x, y, z):= \begin{cases}0, & \text { if } x=y=z, \\ \frac{1}{8}, & \text { if } x, y, z \text { are not pairwise equal } \\ \frac{1}{2}, & \text { and } x, y, z \in\{0,1\} \\ \frac{1}{8}, & \text { otherwise. }\end{cases}
$$


It is easy to verify that $(X, g)$ is a $g-3 p s$ space which is $g$ Hausdorff and $\Sigma$-complete. Note also that $g$ is neither a $G$ metric nor a $S$-metric.

Now, let us consider the map $f: X \rightarrow X$ given by

$$
f x:= \begin{cases}1, & \text { if } x=0 \text { or } x=1 \\ 0, & \text { otherwise }\end{cases}
$$

Obviously, $f$ is $g$-sequentially continuous.

Our results (Theorems 27 and 28) then guarantee the existence and uniqueness of the fixed point $f 1=1$.

\section{Conclusions}

In this work, we pointed out that the results in [6] are obtainable through a metric type space. In addition, a $S$-metric generalizes a $G$-metric only in the case when $G$ is symmetric. We then fill this gap by introducing a new space, namely, a $g-3 p s$ space, which covers a $S$-metric space and also a $G$-metric space in which the symmetric is absent. We also study the underlying topology for this new space in the new direction, totally different from those studied in symmetric and semimetric spaces. We lastly give the sufficient conditions for a fixed point to exist and to be unique.

\section{Acknowledgments}

The authors were supported by the Higher Education Research Promotion and National Research University Project of Thailand, Office of the Higher Education Commission (NRU-CSEC no. 56000508).

\section{References}

[1] S. Gähler, "2-metrische Räume und ihre topologische Struktur," Mathematische Nachrichten, vol. 26, pp. 115-148, 1963.

[2] S. Gähler, "Zur geomitric 2-metriche räume," Revue Roumaine de Mathématiques Pures et Appliquées, vol. 11, pp. 664-669, 1996.

[3] B. C. Dhage, "Generalised metric spaces and mappings with fixed point," Bulletin of the Calcutta Mathematical Society, vol. 84, no. 4, pp. 329-336, 1992.

[4] Z. Mustafa and B. Sims, "A new approach to generalized metric spaces," Journal of Nonlinear and Convex Analysis, vol. 7, no. 2, pp. 289-297, 2006.

[5] S. Sedghi, N. Shobe, and H. Zhou, "A common fixed point theorem in $D^{*}$-metric spaces," Fixed Point Theory and Applications, vol. 2007, Article ID 27906, 13 pages, 2007.

[6] S. Sedghi, N. Shobe, and A. Aliouche, "A generalization of fixed point theorems in S-metric spaces," Matematički Vesnik, vol. 64, no. 3, pp. 258-266, 2012.

[7] Z. Mustafa, H. Obiedat, and F. Awawdeh, "Some fixed point theorem for mapping on complete G-metric spaces," Fixed Point Theory and Applications, vol. 2008, Article ID 189870, 12 pages, 2008.

[8] Z. Mustafa and B. Sims, "Fixed point theorems for contractive mappings in complete G-metric spaces," Fixed Point Theory and Applications, vol. 2009, Article ID 917175, 10 pages, 2009.
[9] H. Aydi, M. Postolache, and W. Shatanawi, "Coupled fixed point results for $(\varphi, \phi)$-weakly contractive mappings in ordered $G$ metric spaces," Computers \& Mathematics with Applications, vol. 63, no. 1, pp. 298-309, 2012.

[10] M. A. Alghamdi and E. Karapınar, " $G-\psi-\varphi$-contractive type mappings in G-metric spaces," Fixed Point Theory and Applications, vol. 123, article 123, 17 pages, 2013.

[11] S. Chandok, W. Sintunavarat, and P. Kumam, "Some coupled common fixed points for a pair of mappings in partially ordered G-metric spaces," Mathematical Sciences, vol. 2013, article 24, 7 pages, 2013.

[12] M. Abbas, W. Sintunavarat, and P. Kumam, "Coupled fixed point of generalized contractive mappings on partially ordered $G$ metric spaces," Fixed Point Theory and Applications, vol. 2012, article 31, 14 pages, 2012.

[13] M. A. Khamsi, "Remarks on cone metric spaces and fixed point theorems of contractive mappings," Fixed Point Theory and Applications, vol. 2010, Article ID 315398, 7 pages, 2010.

[14] I. D. Arandelović and D. J. Kečkić, "Symmetric spaces approach to some fixed point results," Nonlinear Analysis, vol. 75, no. 13, pp. 5157-5168, 2012.

[15] K. Menger, "Untersuchungen über allgemeine," Mathematische Annalen, vol. 100, no. 1, pp. 75-163, 1928.

[16] E. W. Chittenden, "On the equivalence of ecart and voisinage," Transactions of the American Mathematical Society, vol. 18, no. 2, pp. 161-166, 1917.

[17] W. A. Wilson, "On semi-metric spaces," American Journal of Mathematics, vol. 53, no. 2, pp. 361-373, 1931. 


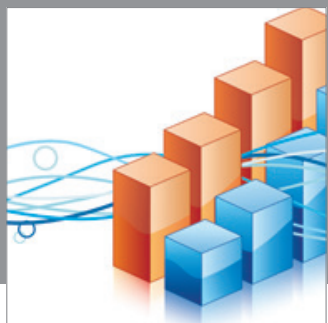

Advances in

Operations Research

mansans

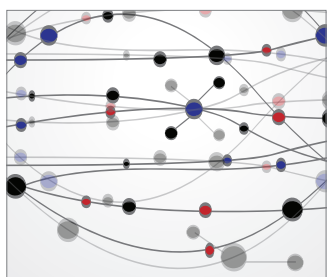

The Scientific World Journal
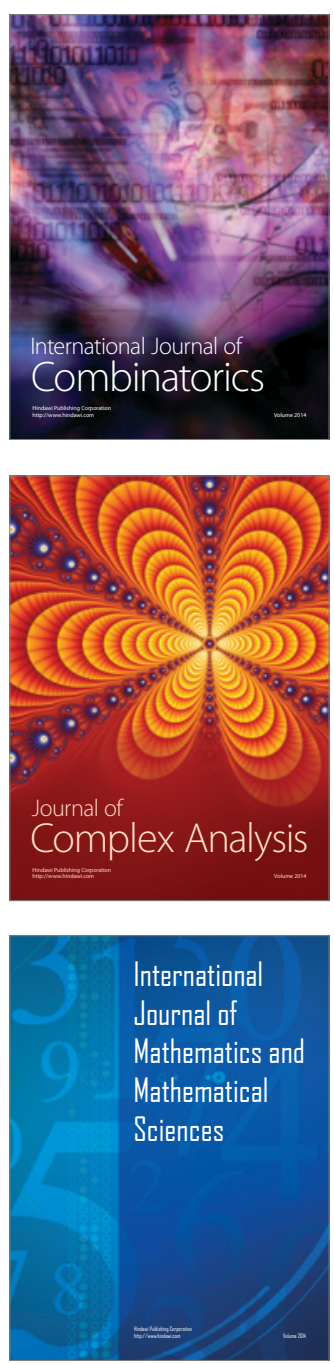
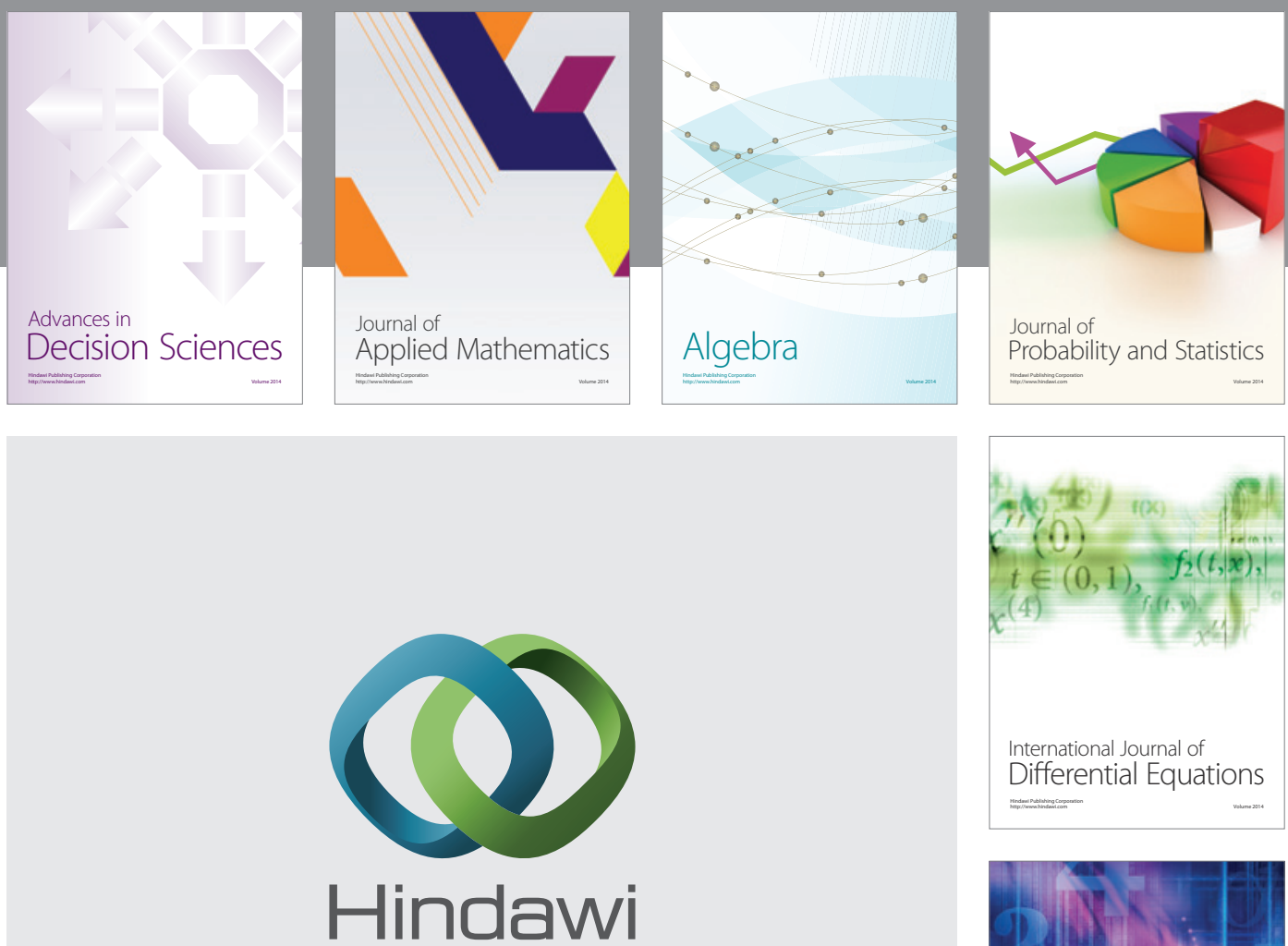

Submit your manuscripts at http://www.hindawi.com
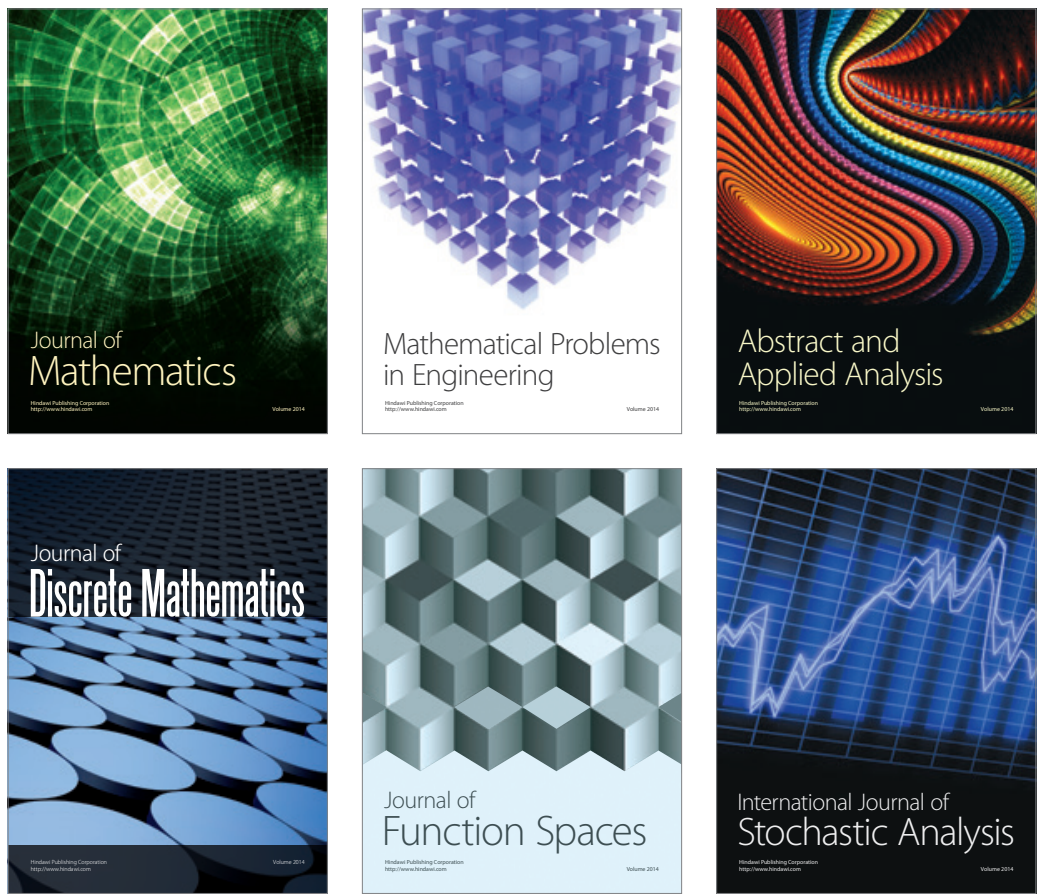

Journal of

Function Spaces

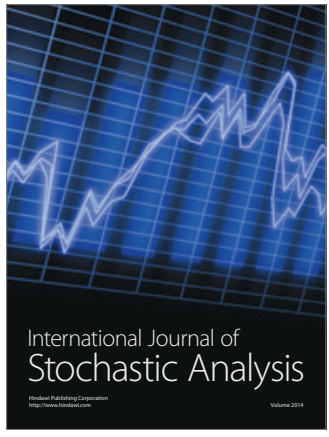

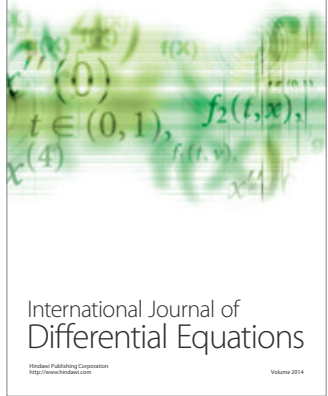
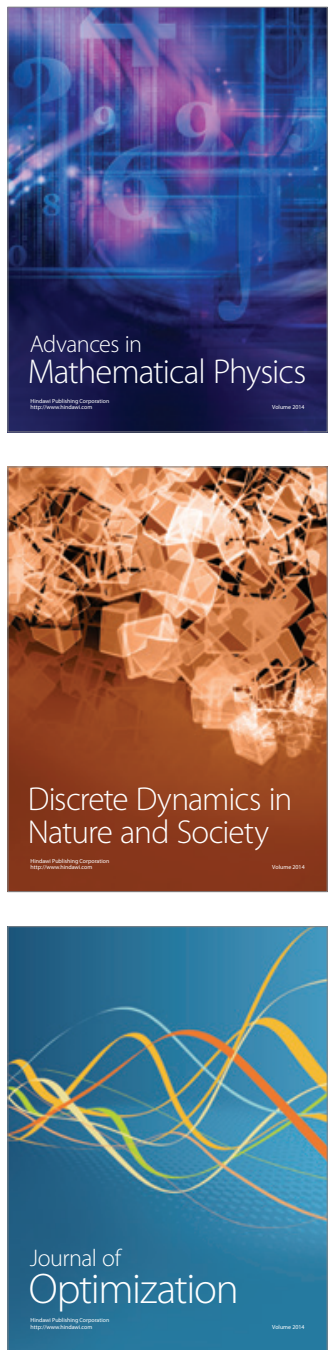